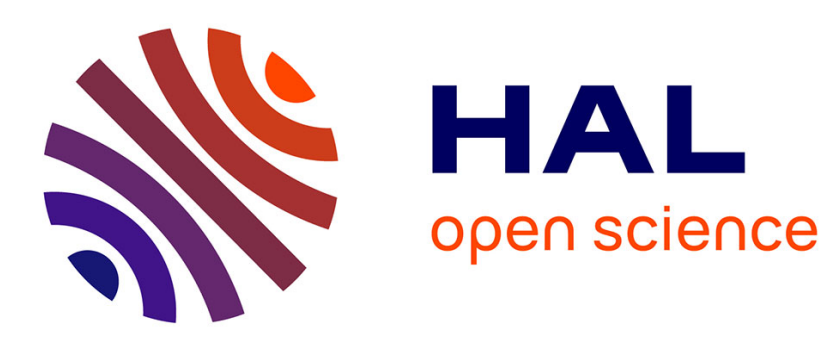

\title{
Sensing technique of dynamic marine mammal's attitude by use of low-cost inertial and magnetic sensors
}

\author{
Hassen Fourati, Noureddine Manamanni, Lissan Afilal, Yves Handrich
}

\section{To cite this version:}

Hassen Fourati, Noureddine Manamanni, Lissan Afilal, Yves Handrich. Sensing technique of dynamic marine mammal's attitude by use of low-cost inertial and magnetic sensors. 8th IFAC Conference on Control Applications in Marine Systems, Sep 2010, Rostock-Warnemünde, Germany. pp. 283-288. hal-00642926

\section{HAL Id: hal-00642926 https://hal.science/hal-00642926}

Submitted on 19 Nov 2011

HAL is a multi-disciplinary open access archive for the deposit and dissemination of scientific research documents, whether they are published or not. The documents may come from teaching and research institutions in France or abroad, or from public or private research centers.
L'archive ouverte pluridisciplinaire HAL, est destinée au dépôt et à la diffusion de documents scientifiques de niveau recherche, publiés ou non, émanant des établissements d'enseignement et de recherche français ou étrangers, des laboratoires publics ou privés. 


\title{
Sensing technique of dynamic marine mammal's attitude by use of low-cost inertial and magnetic sensors
}

\author{
Hassen Fourati****, Noureddine Manamanni*, Lissan Afilal*, Yves Handrich** \\ *CReSTIC, URCA, EA 3804 - Université de Reims Champagne-Ardenne \\ UFR SEN, Moulin de la Housse Bat 12, 51687 Reims Cedex 2 France \\ (e-mail :\{name.surname\}@univ-reims.fr) \\ **Institut Pluridisciplinaire Hubert CURIEN / Département Ecologie, Physiologie et Ethologie \\ UMR 7178 CNRS - Université de Strasbourg, 23 rue du loess \\ 67037 Strasbourg cedex 2,France, (e-mail: yves-jean-handrich@c-strasbourg.fr)
}

\begin{abstract}
This paper presents the design and performance evaluation of a theoretical sensing technique of dynamic marine mammal's attitude. A single sensor pack composed by an Inertial Measurement Unit (IMU), provides all the data required to feed the nonlinear estimator. The proposed algorithm uses complementary data from a 3-axis accelerometer, a 3axis magnetometer and a 3-axis gyroscope. These sensors are mounted in a single tag and appropriately located on the animal. The approach developed here is applied in Bio-logging, an interdisciplinary research area at the intersection of animal behavior and bioengineering. By considering the rigid-body dynamics expressed in quaternion representation, a Complementary Sliding Mode Observer (CSMO) is derived taking advantages from strapdown inertial measurement from gyroscope and vector observations from accelerometer and magnetometer to provide accurate attitude estimates in quaternion representations. The proposed algorithm is based on a multiplicative quaternion correction technique and allows the estimation of large attitude maneuvers of the marine animal. The efficiency of the algorithm herein designed is illustrated through simulations using a theoretical example of animal motion.
\end{abstract}

Keywords: Marine animal's attitude measurement, Complementary Sliding Mode Observer (CSMO), quaternion, MEMS inertial and magnetic sensors, marine biology, navigation, marine logger.

\section{INTRODUCTION}

The attitude estimation of a rigid body is a subject that has attracted a strong interest in many fields such as Walking Robots (Singh and Waldron, 2005), Micro Air Vehicles (Mahony et al., 2008), Marine Vehicles (Vasconcelos et al., 2009) and recently in Bio-logging. The term Bio-logging refers to the use of miniaturized animal-attached tags for logging and/or relaying of data about an animal's movements, behaviour, physiology and/or environment. Biologging has found its origin in the marine environment (Ropert-Coudert et al., 2009; Bograd et al., 2010) and investigations on diving animals represent the core of many studies in this area. Indeed, marine animals are particularly difficult to study during their long foraging trips at sea. However, their need to return periodically to their breeding colony, gives us the opportunity to measure some biological and physical parameters with the use of bio-logger devices (Elkaim et al., 2006; Wilson et al., 2007).

In this paper, the goal is to present a method for body attitude estimation which is suitable for use in marine mammals or birds. The use of MEMS inertial or magnetic sensors in this field is relatively recent. The obvious advantage of these sensors is that we gain access to the third space dimension or orientation, which is the key to a good understanding of the diving strategies observed in these predators (Johnson and Tyack, 2003; Elkaim et al., 2006).
The principle used to track the animal's posture estimates in the afore-mentioned studies consists of using a 3-axis accelerometer and a 3-axis magnetometer, with the reductive assumption that all the movements are static or quasi-static. Consequently, the authors consider that the accelerometer's readings correspond to the earth's gravity measurements in body coordinates, and it therefore becomes possible to estimate the attitude by the resolution of well known Wahba's problem (Wahba, 1965). However, the assumption made above is not valid in all the dynamic situations observed underwater for free-ranging marine animals, e.g. the phases of prey pursuit, and the performance of the attitude estimation is degraded. In (Wilson et al., 2007), the authors assumed that the running mean over a one second interval of total acceleration time is a good estimator of the gravity vector projection. However, this approximation is not valid over time and depends on other parameters such as the animal species and the type of movement it makes. Other work as (Watanabe et al., 2005) is focused on the use of a low-pass filter to extract the gravity projection from the accelerometer outputs. Nevertheless, the tune of the filter's parameters is not easy and can introduce some errors on the attitude in many cases. Our previous works in this area are focused on the proposition of some alternative approaches in order to circumvent the afore-mentioned problems in marine animal's attitude estimation (Fourati et al., 2009; Fourati et al., 2010a; Fourati et al., 2010b). The main idea in these 
papers is to add 3-axis gyroscope measurements to the sensors already used (accelerometer and magnetometer). Nonlinear filters are used to blend data from these sensors in a way to obtain accurate attitude estimates.

This paper continues to explore deeply this area. For this purpose, a Complementary Sliding Mode Observer (CSMO) is proposed to recover the attitude in quaternion representation. It is coupled with a Levenberg Marquardt Algorithm (LMA) and based on measurements from 3-axis gyroscope, 3-axis magnetometer and 3-axis accelerometer. The developed approach combines a strap-down system, based on the time integral of angular velocity, with a LMA that uses vector observations such as earth's magnetic field and gravity vector to compensate the attitude predicted by the gyroscope. It is important to stress that the resulting structure is complementary: high bandwidth rate gyro measurements are combined with low bandwidth vector observations to provide an accurate attitude estimate. The main advantage of this contribution is that only kinematic equation is required, meaning that no knowledge of the animal motion model is needed for the proposed algorithm.

This paper is organized as follows: Section 2 presents the problem formulation and some backgrounds of the rigid body attitude description. Section 3 details the structure of the nonlinear observer. Section 4 is devoted to simulation results that illustrate the proposed observer's performances. Finally, section 5 summarizes the main conclusions of the paper.

\section{MATERIALS AND METHODS}

\subsection{Problem formulation: Bio-Logging application}

The main concern of this study is the development of orientation estimation algorithm for marine mammals animals (considered as rigid bodies). This work belongs to the Bio-logging area and stems from our biologist colleagues' need for a new generation of electronic devices (bio-loggers) and methods which are able to estimate movement patterns with an improved accuracy. The marine model considered in this study is the king penguin (see Fig. 1(a)). This specie is studied in our laboratory for over 20 years. This particular need is motivated by the biologists' need to access to the 3-D posture during motion then calculate the Dynamic Body Acceleration (DBA). This DBA is apparently a good indicator of mechanical work in a moving animal, and is positively correlated with the animal's energy expenditure (Wilson et al., 2007). It is important to note that the king penguin can dives to depths up to $200 \mathrm{~m}$. In these conditions, the pressure can reached 20 bars but no magnetic disturbances can be observed.

In the case of a penguin model, the bird is caught in its breeding colony and the bio-logger is attached to the body as indicated in Fig. 1(b) (near to the gravity center). When the bird's return from a foraging trip at sea, the system is recovered and all the recorded measurements are downloaded. This bio-logger shall comprise: 1) kinematics sensing unit composed of a 3 -axis accelerometer, a 3-axis magnetometer, and a 3-axis gyroscope; 2) pressure sensor; 3) temperature sensor; 4) data acquisition unit; 5) power unit; 6) flash memory. The first prototype of the bio-logger is under conception and still not ready to be deployed on penguin (see
Fig. 2). Before deploying the new logger, the goal in this paper is to be able to convert the raw data (acceleration, angular rate and earth's magnetic field) into relevant information about the rigid body 3-D attitude. The algorithm that will exploit the measurements from this prototype is the main concerns of this work.

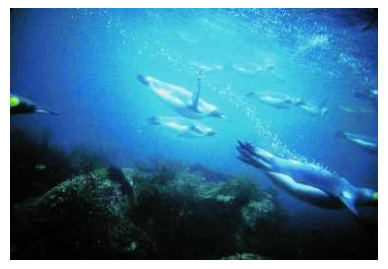

(a)

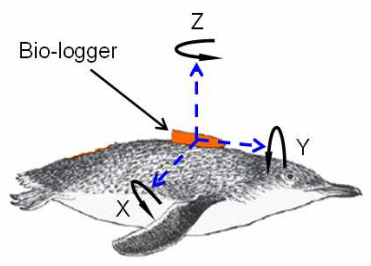

(b)
Fig. 1. (a) King penguin during its dive - (b) Schematic diagram of how the bio-logger is attached to a penguin
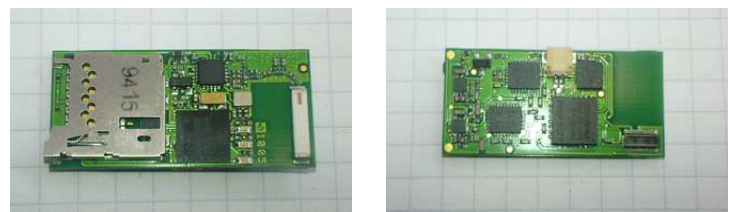

Fig. 2. Exploded view of the overall assembly

\subsection{Rigid body attitude description}

The rigid body attitude estimation problem requires the transformation of measured and computed quantities between body and fixed frames. The frame $B\left(X_{B}, Y_{B}, Z_{B}\right)$ is attached to the rigid body or the animal in our case and the fixed frame $N\left(X_{N}, Y_{N}, Z_{N}\right)$ represents a navigation frame which is tangent to the Earth's surface (Local Tangent Plane, LTP) (see Fig. 3). This local coordinate system is particularly useful to express the attitude of a moving rigid body on the surface of the earth (Grewal et al., 2001). The $X_{N}$-axis points towards to the true north. The $Z_{N}$-axis points towards the interior of the Earth, perpendicular to the reference ellipsoid. The $Y_{N}$-axis completes the right-handed coordinate system, pointing east (NED: North, East, Down). The attitude of a rigid body is based on measurements gained from sensors attached to it. It is important to stress that inertial sensors (3-axis accelerometer and 3-axis gyroscope) and magnetic sensors as 3-axis magnetometer are mounted orthogonally and coincide with to one of the body-platform $B$ (see Fig. 3).

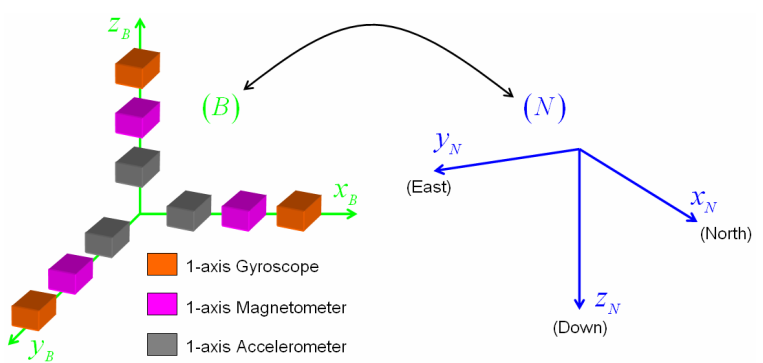

Fig. 3. The body frame (B), the Earth's fixed frame (N) and the configuration of the sensors 
In this paper, we consider the unit quaternion as the mathematical representation of the rigid body attitude between the frames $B$ and $N$. The quaternion, denoted by $q$, is a hyper-complex number of rank 4 such that:

$$
q=q_{0}+q_{1} i+q_{2} j+q_{3} k=\left[\begin{array}{ll}
\cos \frac{\phi}{2} & \vec{u} \sin \frac{\phi}{2}
\end{array}\right]^{T}=\left[\begin{array}{c}
q_{0} \\
q_{\text {vect }}
\end{array}\right]
$$

where $q_{0}, q_{1}, q_{2}$ and $q_{3}$ are real numbers, $i, j$, and $k$ represent the components of the vector $\vec{u}$ (Euler axis), $\phi$ is the rotation angle and $q_{v e c t}=\left[\begin{array}{lll}q_{1} & q_{2} & q_{3}\end{array}\right]^{T} \in \mathfrak{R}^{3 \times 1}$ is the imaginary vector. More details about quaternion algebra can be founded in (Kuipers, 1999).

\section{FILTER DESIGN FOR ATTITUDE ESTIMATION}

Now, our objective is to design a rigid body attitude estimation algorithm based on inertial and magnetic MEMS sensors. The developed approach will be used to track the orientation of the animal specie studied in the project: the king penguin. This work is proposed in order to improve the attitude estimation approaches developed in (Elkaim et al., 2006; Wilson et al., 2007; Watanabe et al., 2005). These works still limited to the static and quasi-static cases of animal's movements. Here, a Complementary Sliding Mode Observer (CSMO) is proposed with a view to do a trade-off between a good short term precision given by rate gyros integration and a reliable long term accuracy provided by accelerometer and magnetometer measurements (attitude observations). It is important to note that other applications and methods (airplanes) add GPS and information and a velocity sensor to improve the attitude measurements accuracy. However, the environmental conditions of our application considered here are limited by certain constraints such as the lack of essential information provided by GPS during the diving activities of these marine predators. Moreover, the velocity sensor is unsuitable to be used on king penguin because it hinders its diving motion.

\subsection{Rigid body kinematic model}

The attitude kinematic equation describes the time rate of change of the attitude $q$, in a quaternion term, as a result of the rigid body angular rates measured by the gyroscope. Therefore, the quaternion derivative that represents the kinematic differential equation is given as in (Vasconcelos et al., 2009) as follows:

$$
\frac{\partial q}{\partial t}=\frac{1}{2} \bar{\omega}_{g} \otimes q
$$

where $\bar{\omega}_{g}=\left[\begin{array}{ll}0 & \omega_{g}\end{array}\right]^{T}$ is the quaternion form of the angular velocity vector $\omega_{g}=\left[\begin{array}{lll}\omega_{g x} & \omega_{g y} & \omega_{g z}\end{array}\right]^{T}$.

The angular velocities measurements $\omega_{g}$ are often corrupted with noises and bias $w(t)$ (Brown and Hwang, 1997). Therefore, including gyroscope disturbances and using the quaternion product $\otimes$ given in (Kuipers, 1999), (2) can be written such as:

$$
\frac{\partial q}{\partial t}=\frac{1}{2}\left[\begin{array}{c}
-q_{\text {vect }}^{T} \\
I_{3 \times 3} q_{0}+\left[q_{\text {vect }}^{\times}\right.
\end{array}\right] \omega_{g}+w(t)
$$

where $\left[q_{\text {vect }}^{\times}\right]$represents the skew-symmetric matrix defined as in (Mahony et al., 2008):

$\left[q_{\text {vect }}^{\times}\right]=\left[\begin{array}{c}q_{1} \\ q_{2} \\ q_{3}\end{array}\right]^{\times}=\left[\begin{array}{ccc}0 & -q_{3} & q_{2} \\ q_{3} & 0 & -q_{1} \\ -q_{2} & q_{1} & 0\end{array}\right]$

$q=\left[\begin{array}{llll}q_{0} & q_{1} & q_{2} & q_{3}\end{array}\right]^{T}$ represents the quaternion vector and $I_{3 \times 3}$ is the identity matrix of dimension 3 .

\subsection{Observer design}

The emphasis of the proposed observer is based on the use of the multiplicative correction technique (Kuipers, 1999):

$q^{\prime}=\delta q \otimes q$

Quaternion multiplication is used in (5) to correct the quaternion calculation process. This equation is usually used in quaternion algebra to update a quaternion (Kuipers, 1999). $\delta q$ is the correction term which is a function of the measurement error.

To perform measurement update of the quaternion estimates, (5) can be transformed into the following form:

$\hat{q}^{+}=\delta \hat{q}^{+} \otimes \hat{q}^{-}$

where $\hat{q}^{+}$and $\hat{q}^{-}$represent the post and pre-update values of the quaternion estimates, respectively.

Finally, the CSMO can be designed by using (6) with the choice of a sliding manifold based on the error quaternion. The proposed observer is given as follows:

$$
\begin{aligned}
& \frac{\partial \hat{q}}{\partial t}=\delta_{K_{1}} \otimes \delta_{K_{2}} \otimes \frac{1}{2}\left[\begin{array}{c}
-\hat{q}_{\text {vect }}^{T} \\
I_{3 \times 3} \hat{q}_{0}+\left[\hat{q}_{\text {vect }}^{\times}\right]
\end{array}\right] \omega_{g} \\
& \left\{\delta_{K_{1}}=\frac{1}{\left\|\chi_{1}\right\|} \chi_{1} \quad ; \quad \delta_{K_{2}}=\frac{1}{\left\|\chi_{2}\right\|} \chi_{2}\right. \\
& \chi_{1}=K_{1}\left[\begin{array}{ll}
1 & \text { sat }\left[\frac{q_{e, v e c t}}{\rho}\right]
\end{array}\right]^{T} ; \quad \chi_{2}=K_{2}\left[\begin{array}{ll}
1 & q_{e, v e c t}
\end{array}\right]^{T}
\end{aligned}
$$

where $\hat{q}$ is the estimated quaternion at time $t$ and $\left[\hat{q}_{v e c t}^{\times}\right]$is defined as in (4). $\delta_{K_{1}}$ represents the switching correction term and $\delta_{K_{2}}$ is the linear correction term. In order to perform the quaternion multiplication, each correction term should be converted into a quaternion. This conversion is obtained using the forced normalization method given in (Deutschmann et al., 1992). The observer gains $K_{1}$ and $K_{2}$ can be written such as: 


$$
K_{1}=\left[\begin{array}{cccc}
1 & 0 & 0 & 0 \\
0 & k_{1} & 0 & 0 \\
0 & 0 & k_{2} & 0 \\
0 & 0 & 0 & k_{3}
\end{array}\right] ; K_{2}=\left[\begin{array}{cccc}
1 & 0 & 0 & 0 \\
0 & k_{4} & 0 & 0 \\
0 & 0 & k_{5} & 0 \\
0 & 0 & 0 & k_{6}
\end{array}\right]
$$

The scalar parts of $\chi_{1}$ and $\chi_{2}$ are chosen very close to unit since the incremental quaternion corresponds to a small angle rotation (Elkaim et al., 2006). The saturation function sat is used to avoid the high frequency chattering behavior around the sliding surface:

$$
\operatorname{sat}\left(\frac{q_{e, v e c t}}{\rho}\right)= \begin{cases}1 & q_{e, \text { vect }} \leq \rho \\ \frac{q_{e, v e c t}}{\rho} & \left|q_{e, \text { vect }}\right| \leq|\rho| \\ -1 & q_{e, \text { vect }} \geq \rho\end{cases}
$$

The parameter $\rho$ is the sliding surface boundary layer. It determines the sliding behavior in the vicinity of $q_{e, \text { vect }}=0$. Note that $q_{e, v e c t}$ is the imaginary vector of the error quaternion $q_{e} \cdot q_{e}$ measures the discrepancy between the complementary estimated quaternion $\overline{\hat{q}}$ and the measured attitude $q_{m}$, that is:

$$
q_{e}=q_{m} \otimes \overline{\hat{q}}=\left[\begin{array}{ll}
q_{e 0} & q_{e, v e c t}
\end{array}\right]^{T}
$$

$q_{m}$ is called also attitude observation and may be determined from two vector observations, such as those obtained by magnetometers and accelerometers (Fourati et al., 2010b). The problem of determining $q_{m}$ using vector measurements is known in the literature as Wahba's problem (Wahba, 1965) and the solution is proposed in the section 2.3.

To preserve the unit quaternion norm, the estimated quaternion $\hat{q}$ in (7) must be normalized to. Normalization is obtained such as:

$$
\hat{q}_{\text {norm }}=\frac{\hat{q}}{\|\hat{q}\|}
$$

\subsection{Optimal determination of attitude observation $q_{m}$}

The magnetometer provides the magnetic field vector in the frame $B$. This quantity is locally constant in the frame $N$ and therefore it represents the first vector observation. For sufficiently low frequency bandwidths, the gravitational field also dominates the accelerometer measurements, as discussed in (Mahony et al., 2008). This would provide a second vector observation. We propose to use a Levenberg Marquardt Algorithm (LMA) to recover measurements of attitude observations $q_{m}$, by using a 3 -axis accelerometer and a 3axis magnetometer (Fourati et al., 2010a).

The 3-axis accelerometer measures the specific force $f$ in the body frame $B$ as follows:

$$
f=\left[\begin{array}{lll}
f_{x} & f_{y} & f_{z}
\end{array}\right]^{T}=M_{N}^{B}(q)[a+g]+\delta_{f}
$$

where $g=\left[\begin{array}{lll}0 & 0 & 9.81\end{array}\right]^{T}$ and $a \in \mathfrak{R}^{3}$ represent respectively the gravity vector and the inertial acceleration of the body, expressed in the navigation frame $N . \delta_{f} \in \mathfrak{R}^{3}$ is a white Gaussian noise. The rotation matrix $M_{N}^{B}(q)$ is expressed as:

$$
M_{N}^{B}(q)=\left[\begin{array}{ccc}
2 q_{0}^{2}+2 q_{1}^{2}-1 & 2\left(q_{1} q_{2}+q_{0} q_{3}\right) & 2\left(q_{1} q_{3}-q_{0} q_{2}\right) \\
2\left(q_{1} q_{2}-q_{0} q_{3}\right) & 2 q_{0}^{2}+2 q_{2}^{2}-1 & 2\left(q_{0} q_{1}+q_{2} q_{3}\right) \\
2\left(q_{0} q_{2}+q_{1} q_{3}\right) & 2\left(q_{2} q_{3}-q_{0} q_{1}\right) & 2 q_{0}^{2}+2 q_{3}^{2}-1
\end{array}\right]
$$

The 3-axis magnetometer gives the direction of the Earth's magnetic field in the body frame $B$ as follows:

$h=\left[\begin{array}{lll}h_{x} & h_{y} & h_{z}\end{array}\right]^{T}=M_{N}^{B}(q) m+\delta_{h}$

where

$m=\left[\begin{array}{lll}m_{x} & 0 & m_{z}\end{array}\right]^{T}=[\|m\| \cos (\theta) \quad 0 \quad\|m\| \sin (\theta)]^{T}$

$m$ represents the magnetic field vector measured in the navigation frame $N$. The theoretical model of the magnetic field nearest to reality considers a magnetic field vector with an inclination angle $\theta=60^{\circ}$ and a norm vector $\|m\|=0.5$ Gauss (Astrosurf, 2010). $\delta_{h} \in \mathfrak{R}^{3}$ is a white Gaussian noise and the rotation matrix $M_{N}^{B}(q)$ is as expressed in (13). The LMA is an estimator that uses the Earth's magnetic field $m$ and the gravity vector $g$ as vector observations to deduce the attitude $q_{m}$ (Fourati et al., 2009). This algorithm is based on the followings steps:

1) Measure accelerometer and magnetometer readings $f$ and $h$, respectively.

2) Calculate $\hat{f}^{N}=\hat{q} \otimes f \otimes \overline{\hat{q}}$ and do the same for $\hat{h}^{N}$. Note that $\hat{f}^{N}$ and $\hat{h}^{N}$ represent the estimated acceleration and magnetic field values in the navigation frame $N$.

Note also that $\overline{\hat{q}}=\left[\begin{array}{llll}\hat{q}_{0} & -\hat{q}_{1} & -\hat{q}_{2} & -\hat{q}_{3}\end{array}\right]^{T}$ represents the quaternion inverse of $\hat{q}$.

3) Calculate the navigation error $\partial f=g-\hat{f}^{N}$ and do the same for $\partial h=m-\hat{h}^{N}$ in order to form $z=\left[\begin{array}{ll}\partial h & \partial f\end{array}\right]^{T}$.

4) Calculate the Jacobian matrix:

$$
J=-2\left[\left[\left[\hat{h}^{N}\right]^{\times}\right]^{T}\left[\left[\hat{f}^{N}\right]^{\times}\right]^{T}\right]^{T}
$$

5) Calculate the pseudo-inverse $O^{*}=\left[J^{T} J+\lambda I_{3 \times 3}\right]^{-1} J^{T}$.

6) Calculate the quaternion error such as: $q_{e}(t)=\alpha O^{*} z, \alpha$ is a smooth parameter chosen between 0 and 1 (Elkaim et al., 2006).

7) Calculate $q_{m}$ such as: $q_{m}(t)=\hat{q}(t) \otimes\left[\begin{array}{ll}1 & q_{e}(t)\end{array}\right]^{T}$.

Fig. 4 illustrates the attitude estimation algorithm performed by the proposed nonlinear observer. 


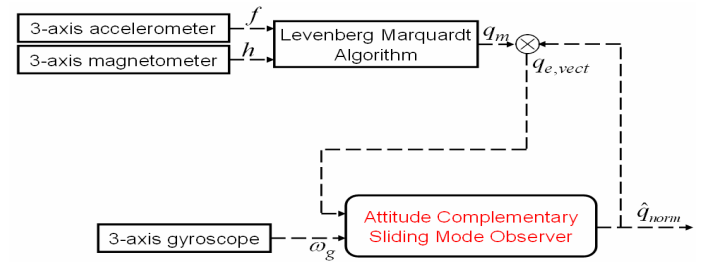

Fig. 4. Scheme of the estimation algorithm

A frequency analysis of inertial and magnetic sensors shows that signals coming from the accelerometermagnetometer pair and signals from the gyroscope have a complementary frequency spectrum (Brown and Hwang, 1997). Therefore, the resulting structure of the sliding mode observer (7) blends the low frequency region (low bandwidth) of the accelerometer and magnetometer data, where the attitude is typically more accurate, with the high frequency region (high bandwidth) of the gyroscope data, where the integration of the angular velocity yields better attitude estimates (Vasconcelos et al., 2009).

\section{THEORETICAL SIMULATIONS AND RESULTS}

This section aims to illustrate the performance of the CSMO in estimating the rigid body attitude based on inertial and magnetic measurements. Some numerical simulations were carried out according to the conditions of our final application on marine animals (the case of the king penguin). Therefore, one considers that the triad of sensors (3-axis accelerometer, 3 axis magnetometer and 3-axis gyroscope) is attached to the animal body (see Fig. 5). The instrument axes are nominally aligned with the body-platform axes.

The simulations under Matlab begin with the generation of theoretical measurements of angular rates, specific forces and magnetic fields taken respectively from gyroscope, accelerometer and magnetometer, during the animal's movement (see Fig. 5). To represent the sensor imperfections, an additional random zero-mean white Gaussian noise was considered for all measurements (see Table 1). The sampling rate was chosen as $100 \mathrm{~Hz}$ for all measurements $(0.01 \mathrm{sec})$. The kinematic equation (3), that exploits simulated angular rates, is solved to obtain a theoretical example of continuous time motion in quaternion representation. The obtained posture is used in the simulations as a reference for comparison with the proposed observer. The boundary layer thickness is set to $\delta=3.10^{-4}$.

The constants $k_{1}, k_{2}, k_{3}, k_{4}, k_{5}$, and $k_{6}$ in the observer gains $K_{1}$ and $K_{2}$ (equation (8)) that guarantee convergent estimates, are set according to the considered sensor noise levels and sampling rate such as follows: $k_{1}=k_{2}=k_{3}=10^{-3}$ and $k_{4}=k_{5}=k_{6}=9.10^{-4}$.

Table 1. Characteristics of the various noises for the sensors measurements

\begin{tabular}{|c|c|c|c|}
\hline Sensors & Parameters & $\begin{array}{c}\text { Standard } \\
\text { deviations }\end{array}$ & Units \\
\hline Accelerometer & $\delta_{f}$ & 0.4 & $\mathrm{~m} / \mathrm{s}^{2}$ \\
\hline Magnetometer & $\delta_{h}$ & 0.4 & $\mathrm{Gauss}$ \\
\hline Gyroscope & $\delta_{g}$ & 0.4 & $\mathrm{rad} / \mathrm{s}$ \\
\hline
\end{tabular}

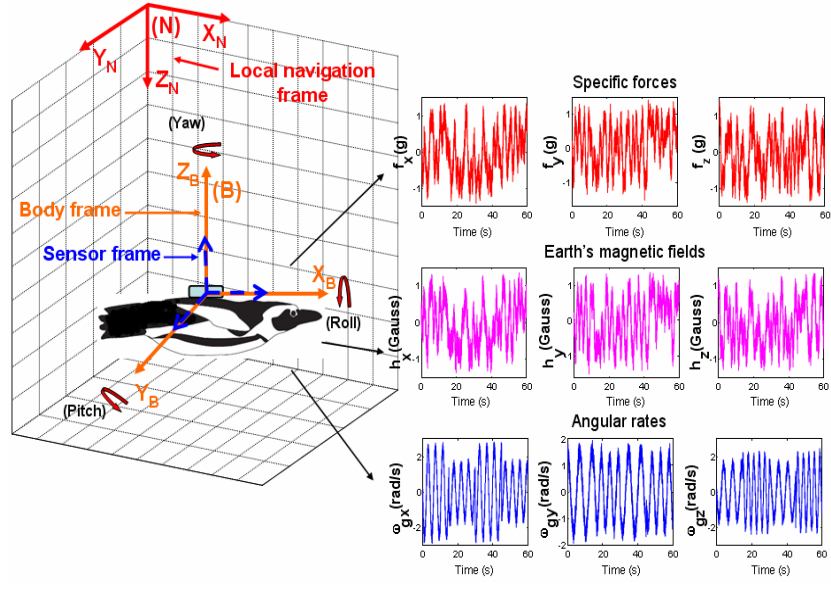

Fig. 5. Simulation using a theoretical representation of the final application

In this set of simulations, the theoretical states of the model (the components of the quaternion $q$ ) are initialized with different random values as well as those of the CSMO. These conditions are summarized such as:

$$
q\left(t_{0}\right)=\left[\begin{array}{llll}
1 & 0 & 0 & 0
\end{array}\right]^{T} \quad ; \quad \hat{q}\left(t_{0}\right)=\left[\begin{array}{llll}
0.2 & 0.5 & 0.7 & 0.3
\end{array}\right]^{T}
$$

Notice that this choice allows us to illustrate the convergence of the observer even though it was initialized far from the actual states. In order to evaluate the overall performance of the attitude estimation, we plotted the time history evolution of the estimation errors on the quaternion. Fig. 6 depicts the convergence of these errors towards zero during the simulated motion. For more clarity, two scales are used, one for periods between 0 and 5 seconds and another for periods lasting longer than 5 seconds. This zoom illustrates the convergence behavior early in the time course and shows the precision obtained after convergence as clearly as possible. Despite the fact that the CSMO and the theoretical model of the quaternion were initialized with different initial conditions, one can note that the estimated quaternion rapidly converges towards the theoretical values. The same performance was obtained when using different sets of initial conditions.
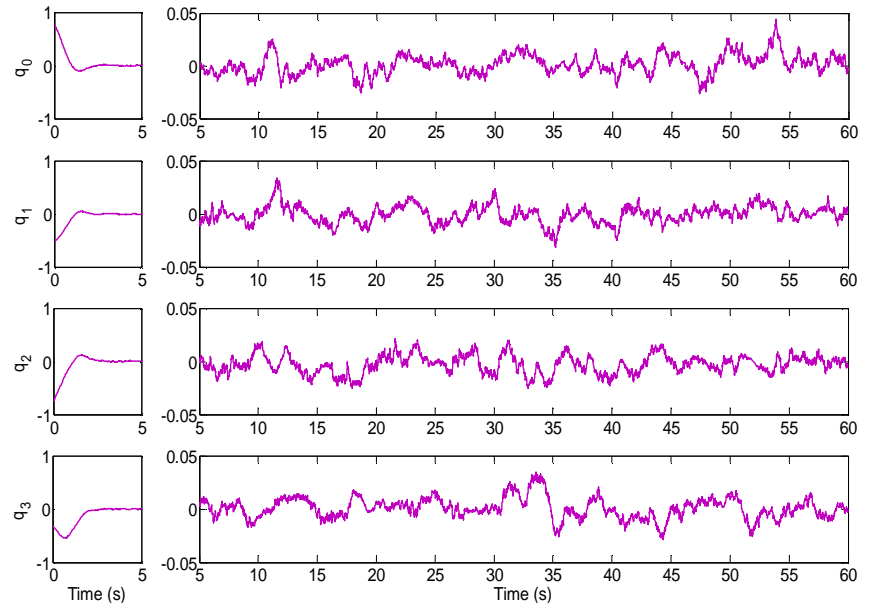

Fig. 6. Quaternion estimation errors using CSMO (with a zoom between 5 and $60 \mathrm{sec}$ ) 
It is clear that the domain of attraction seems to be large enough, since the states are initialized far from their true values and the observer converges quite fast. The nonlinear observer copes well with the large noises introduced in the gyroscope, accelerometer and magnetometer measurements. In order to show the improvements made by the CSMO in the Bio-logging field, we depict in Fig. 7 the quaternion estimation errors resulting from the method developed in (Elkaim et al., 2006) for the same area. It is important to note that the rate of errors committed by this approach is very large. Indeed, this approach fails to track the desired attitude because the noises from motion accelerations and vibrations affect the accelerometer measurements. Hence, the obtained results show that the CSMO is an efficient method for improving the attitude estimation quality.
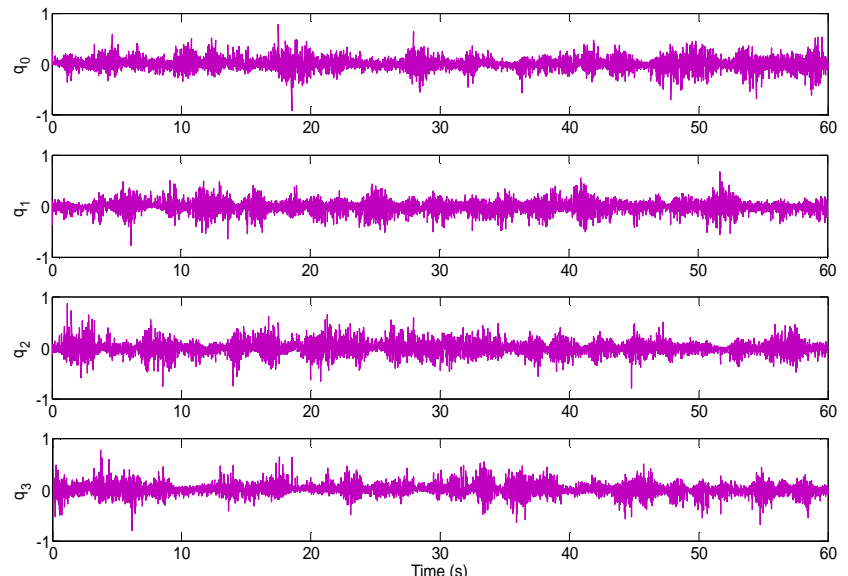

Fig. 7. Quaternion estimation errors using method (Elkaim et al., 2006)

\section{CONCLUSIONS}

This paper focuses on the development of an alternative method to recover the full orientation and attitude of marine animals. The application in mind is that of Bio-logging. A Complementary Sliding Mode Observer (CSMO) is designed based on the complementary filtering theory and the multiplicative quaternion correction technique. By considering the rigid body kinematic model, the CSMO is proposed with a view to do a trade-off between a good short term precision given by rate gyros integration and a reliable long term accuracy provided by accelerometers and magnetometers measurements (attitude observations). The efficiency of the proposed approach is shown with simulation tests using a theoretical example of animal motion. Future works concern the experimental evaluation of this approach in real world application, especially on the king penguins.

\section{AKNOWLEDGMENTS}

The authors would like to thank both of Alsace and Champagne-Ardenne regions within the framework of the project (NaviMeles) for their financial support.

\section{REFERENCES}

Astrosurf, (2010). Available: http://www.astrosurf.com. Bograd, S. J., Block, B. A., Costa, D. P., and Godley, B. J. (2010). Biologging technologies: new tools for conservation. Introduction, Endangered Species Research, 10, 1-7.

Brown, R.G., and Hwang, P.Y.C. (1997). Introduction to Random Signal and Applied Kalman Filtering, $3^{\text {rd }}$ Ed. New York: John Wiley.

Deutschmann, J., Bar-Itzhack, I., and Galal, K. (1992). Quaternion normalization in spacecraft attitude determination, AIAA Astrodynamics Conference, 27-37.

Elkaim, G.H., Decker, E.B., Oliver, G., and Wright, B. (2006). Marine Mammal Marker (MAMMARK) dead reckoning sensor for In-Situ environmental monitoring, in Proc. IEEE Position, Loc. and Navigation Symposium, Monterey, 976-987.

Fourati, H., Manamanni, N., Afilal, L., and Handrich, Y. (2009). A rigid body attitude estimation for Bio-logging application: A quaternion-based nonlinear filter approach, IEEE/RSJ International Conference on Intelligent Robots and Systems IROS'09, St. Louis, USA, 558-563.

Fourati, H., Manamanni, N., Afilal, L., and Handrich, Y. (2010a). A nonlinear filtering approach for the attitude and Dynamic Body Acceleration estimation based on inertial and magnetic sensors: Bio-logging application, IEEE Sensors Journal, accepted, in press.

Fourati, H., Manamanni, N., Afilal, L., and Handrich, Y. (2010b). Posture and body acceleration tracking by inertial and magnetic sensing: Application in behavioural analysis of free-ranging animals, Biomedical Signal Processing and Control, doi: 10.1016/j.bspc.2010.06.004.

Grewal, M. S., Weill. L. R., and Andrews, A. P. (2001). Global positioning systems, inertial navigation and integration, John Wiley \& Sons, Inc.

Johnson, M.P., and Tyack. P.L. (2003). A Digital Acoustic Recording Tag for Measuring the Response of Wild Marine Mammals to Sound. IEEE Journal of Oceanic Engineering, 28(1), 3-12.

Mahony, R., Hamel. T., and Pflimlin, J. M. (2008). Nonlinear complementary filters on the special orthogonal group, IEEE Transactions on Automatic Control, 53(5), 12031218.

Ropert-Coudert, Y., Beaulieu, M., Hanuise, N., and Kato, A. (2009). Diving into the world of biologging. Endangered Species Research, 10, 21-27.

Singh, S. P. N., and Waldron, K. J. (2005). Attitude estimation for dynamic legged locomotion using range and inertial sensors, IEEE International Conference on Robotics and Automation, Barcelona, Spain, 1663-1668.

Vasconcelos, J. F., Silvestre, C., Oliveira, P., Batista, P., and Cardeira, B. (2009). Discrete time-varying attitude complementary filter, IEEE American Control Conference, St. Louis, USA, 4056-4061.

Wahba, G. (1965). A least squares estimate of spacecraft attitude, SIAM, Review, 7(3), 409.

Watanabe, S., Isawa, M., Kato, A., Coudert, Y., and Naito, Y. (2005). A new technique for monitoring the behaviour of terrestrial animals; a case study with the domestic cat, Appl Anim Behav Sci, 94, 117-131.

Wilson, R., Shepard, E. L. C., and Liebsch, N. (2008). Prying into the intimate details of animal lives: use of a daily diary on animals. Endangered Species Research, 4, 123137. 